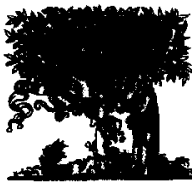

ELSEVIER

\title{
Editorial
}

\section{Hepatitis C anno 1997}

\author{
S.W. Schalm * \\ Department of Hepatogastroenterology and Internal Medicine, Erasmus University Hospital-Dijkzigl, PO Box 2040, 3000 CA Rotterdum, \\ Netherlands
}

Rcceived 26 August 1997; accepted 2 September 1997

\section{Introduction}

For hepatitis C, the year 1997 is characterized by consensus reports from France and the USA [1,2]. Ten years after the observation that therapy with $\alpha$-interferon can induce biochemical remission in chronic active non- $A$, non-B hepatitis, 7 years after the discovery of hepatitis $\mathrm{C}$ virus (HCV) as the aetiological agent of chronic non-A, non-B hepatitis, there was a broadly felt need to establish consensus on methods for diagnosis and therapy and to explore the public health implication of a chronic disease with a prevalence of $0.2-1.2 \%$ in NW Europe and North America, and a rising cause of liver transplantation.

What were the issues? In few areas of hepatology have findings on the sensitivity and specificity of diagnostic tests, and the results of intervention studies been so homogenous as in HCV. Despite this solid base, opinions on strategies and management voiced by physicians who considered themselves experts have been distressingly divergent [3]. The key questions, listed below, were central to the consensus meetings in France and in the USA.

- What is the natural history of hepatitis C?

- What is the most appropriate approach to diagnose and monitor patients?

\footnotetext{
${ }^{*}$ Tel: + 3110 4635942; fax: +31 10 4365916; e-mail: schalm@inw2.azr.nl
}

- What recommendations can be made to patients to prevent transmission?

- Which patients should be treated?

- What is the most effective approach to therapy?

The French report also specifically addresses the question of screening the general population or risk groups, such as recipients of blood products prior to 1991, and past or present users of intravenous drugs.

Remarkably, very similar consensus statements with clear-cut recommendations have appeared. It is of interest that both consensus statements were formulated by a panel of experienced scientists of various disciplines without expertise in hepatitis $\mathrm{C}$, after hearing the evidence provided by a series of hepatitis $\mathrm{C}$ experts.

In this issue of the Netherlands Journal of Medicine, two Dutch experts on hepatitis $C$ review the progress on chronic hepatitis $\mathrm{C}$ virus disease in the last 5 years [4]. There are several discrepancies between the consensus reports and the Dutch review.

\section{The natural history of hepatitis $C$}

The statement in the Dutch review article that "of those infected probably $60 \%$ and perhaps up to $90 \%$ develop chronic infection resulting in cirrhosis in $20-50 \%$ after a mean duration of about 20 years" may be not so much different from the statement in the American Report, which mentions development 
of cirrhosis in at least $20 \%$ within two decades of onset of infection. However, the American report. mitigating this statement, also reads "chronic hepatitis $\mathrm{C}$ is typically an insidious process, progressing, if at all, at a slow rate without symptoms or physical signs in the majority of patients during the first two decades after infection". In contrast, the Dutch review article underlines their original statement with the comment that the rate of progression to cirrhosis is diverging and may take less than 5 years, thereby stressing the severity of the disease. Much of the uncertainty of the natural history of chronic hepatitis $\mathrm{C}$ has been solved by a French retrospective study in 2235 patients [5]. Three independent factors associated with progressive liver disease were identified: age of more than 40 years at the time of infection; daily use of $50 \mathrm{~g}$ alcohol or more; and male gender. The group of cirrhosis within 30 years $(33 \%$ of the total group), was characterized by males and consumers of alcohol; the group unlikely to develop cirrhosis in 50 years (30\% of the total group), was predominantly women, infected at a young age and abstainers from alcohol.

\section{The diagnosis of hepatitis $\mathrm{C}$}

The paragraph on ELISA methods in the Dutch review suggests both false-positive and false-negative results in a clinically relevant number of patients, in contrast to the French and the American texts stating that scientific and medico-economic data allow, without hesitation, the practice of testing for $\mathrm{HCV}$ by a single EIA test (second or third generation) in the majority of individuals. For low-risk individuals, such as blood donors, a negative EIA is sufficient to rule out infection. In patients presenting with liver disease, a positive EIA test is sufficient to diagnose hepatitis $\mathrm{C}$ infection; a qualitative $\mathrm{HCV}$ RNA test can be used for confirmation. Low risk individuals with a positive EIA test should undergo supplemental testing. The practice of confirming positive results by RIBA has now become a matter of debate; confirmation can also be made by anti-HCV testing in a second blood sample, supplemented by HCV RNA testing, if indicated, and if reliable laboratory facilities are accessible.

\section{Recommendations to prevent transmission}

Both consensus reports mention the very low risk of sexual transmission (the Dutch review mentions 6-25\%) and advise no special measures for stable relationships except in the case of genital lesions or menstruation. For individuals with multiple sexual partners, safe-sexual practices, including the use of latex condoms, are strongly encouraged as a general approach of avoiding disease transmission.

\section{Indications for interferon therapy}

The Dutch review recommends antiviral therapy for all individuals with detectable HCV RNA and a liver biopsy showing chronic hepatitis (see Fig. 1 in review).

The consensus reports do not recommend antiviral therapy for individuals with normal ALT (about 30\% of the index population) nor for patients with cirrhosis (about $20 \%$ ). Only patients with chronic hepatitis, elevated ALT and histologically at least moderate lobular or piecemeal necrosis are candidates for therapy.

\section{Most effective approach to therapy}

The standard intention-to-treat regimen is 3 million units of $\alpha$-interferon thrice weekly for 12 months. However, patients without ALT normalisation after 3 months (about $40 \%$ ) or those with initial ALT normalisation, but 'breakthrough' (about 25\%), have virtually no chance of a sustained response and should discontinue interferon therapy, according to the consensus reports. The Dutch review mentions several options, but is less specific with regard to the standard approach anno 1997.

\section{Discussion}

Developments in the management of viral diseases are rapid. Dutch investigators have contributed and are still contributing substantially to this exciting field. In this setting, it is only natural and even essential that experts explore different approaches in their clinical research. When it comes to updating the 
medical profession, balanced guidelines formulated by experienced scientists not directly involved in the field, like the French and American consensus reports, may be preferable to the personal opinions of experts in the field, in view of the divergent results registered by an epidemiological survey [3].

\section{References}

[1] Hépatite C: dépistage et trâitement. Gastroenterol Clin Biol 1997;20:S202-211.
[2] National Institutes of Health Consensus Development Conference Panel Statement: Management of hepatitis C. Hepatology 1997;26(Suppl. 1):2S-10S.

[3] Nolpas B. Delaroques-Astagneau E, Le Bihan C, Drucker J, Desenclos JC. Medical practices of EASL members regarding HCV infection. J Hepatol 1997;26(Suppl. 1):S78.

[4] Stadhouders, PHGM, Cooreman, MP, Chronic Hepatitis C virus disease. An evaluation of procedures for diagnosis and treatment. Neth J Med, in press.

[5] OBSVIRC, METAVIR, CLINIVIR and DOSCVIR groups, Poynard T, Bedossa P, Opolon P. Natural history of liver fibrosis: progression in patients with chronic hepatitis $C$. Lancet 1997;349:825-832. 